\title{
Substance Index for Volume 63
}

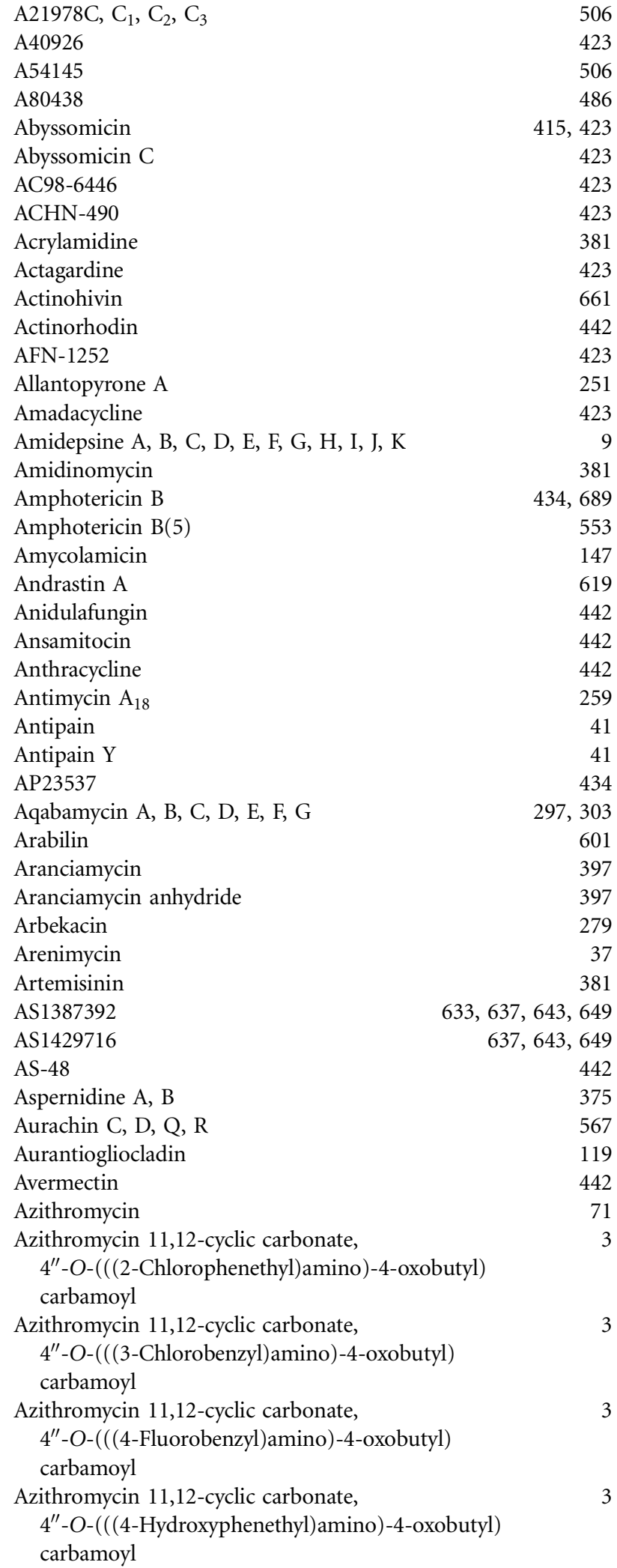

Azithromycin 11,12-cyclic carbonate, 3 $4^{\prime \prime}$-O-(((4-Methoxybenzyl)amino)-4-oxobutyl) carbamoyl

Azithromycin 11,12-cyclic carbonate, $4^{\prime \prime}-O-((($ Benzyl)amino)-4-oxobutyl)carbamoyl

Azithromycin 11,12-cyclic carbonate, $4^{\prime \prime}$-O-(((Pentyl)amino)-4-oxobutyl)carbamoyl

Azithromycin 11,12-cyclic carbonate, $4^{\prime \prime}$-O-(((Phenethyl)amino)-4-oxobutyl)carbamoyl

Bafilomycin A1 359

Bafilomycin $A_{1}, 17,18$-dehydro-19,21-di-O-methyl- 595 24-demethyl-

Bafilomycin $A_{1}$, 19,21-di-O-methyl-24-demethyl- 595

Bafilomycin $A_{1}$, 21-O-methyl-24-demethyl- 595

Bafilomycin $A_{1}$, 24-demethyl- $\quad 595$

Bafilomycin C1, C2 359

Bafilomycin D 359

Bafilomycin D, 24-demethyl- $\quad 595$

BAL-30072 423

Bassianolide $\quad 77$

BC-3205 423

BD-12 381

Beauvericin $\quad 77$

Bellenamine $\quad 381$

Benzo[a]naphtacene-2-carboxylic acid, $\quad 709$

4,7,11,12-tetrahydro-1,5,6,9,14-pentahydroxy-

8,13-dioxo-3-methyl-10-ethyl

Betulin derivatives 123

$1 \mathrm{H}, 1^{\prime} \mathrm{H}-\left[3,3^{\prime}\right]$ biindolyl $\quad 681$

Bishomononactic acid 524

Bispolide $\quad 275$

Bispolide derivatives $\quad 275$

Blasticidin A, S $\quad 309$

Bleomycin 442

Bluensomycin 442

Brasiliquinone B $\quad 545$

Bromochlorogentisylquinone A, B 199

$\begin{array}{ll}\text { BY-81 } & 381\end{array}$

C-1027 482

Caboxamycin $\quad 415$

Calpinactam $\quad 183$

Candicidin 434

Caripyrin 285

CB-182804 423

CCl779 434

Ceftaroline 423

Ceftizoxime 533

Ceftobiprole $\quad 423$

CEM-101 423

Cephalosporin C, deacetoxy $\quad 442$

Cephamycin C 442

8-Chloro-3', $4^{\prime}, 5,7$-tetrahydroxyisoflavone 619

Chloroquine 381 
$\begin{array}{ll}\text { Cisplatin } & 530\end{array}$

Citreoviridin 559

Clavulanic acid 442

Clofazimine $\quad 579$

Coelomycin $\quad 512$

Colistin 51

CXA-101 423

Cyclamidomycin $\quad 381$

Cyclo-(Pro-Ile) 699

Cyclo-(Pro-Leu) 699

Cyclosporine 434

Cyclosporine A $\quad 415$

Cyperusol C 245

Dactinomycin 442

Dalbavancin 423

Daptomycin 279, 415, 423, 506

Decalpenic acid 703

Deferolimus $\quad 434$

Delafloxacin $\quad 423$

Deoxyactagardine B $\quad 351$

Difimicin 423

$8 \alpha, 13$-Dihydroxy-marasm-5-oic acid $\gamma$-lactone $\quad 575$

Dinactin 524

Doripenem $\quad 631$

Doxorubicin $\quad 442$

Doxycycline $\quad 693$

Dysideanin A, B 699

ECO-02301, -0501 423

$\begin{array}{ll}\text { Eflornithine } & 381,579\end{array}$

Enniatin A, A1, B, B1, D, F

Enomicin A, methyl 442

(22E,24R)-5 $\alpha, 8 \alpha$-epidioxyergosta-6,22- $\quad 575$

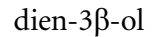

Epothilone A, A9, B, C, M, N 571

Equisetin 195

Eremomycin, chloro- $\quad 423$

$\begin{array}{ll}\text { Erythromycin } & 279,442\end{array}$

Erythromycin A $\quad 442$

Erythromycin A, 6,11-di-O-methyl, 4" - 343

carbamates

Erythromycin A, 6,11-di-O-methyl-2'-O-acetyl 343

Erythromycin A, 6,11-di-O-methyl-2'-O-acetyl-4" - 343

O-acylimidazolyl

Erythromycin A, 6,11-di-O-methyl-4"-O( $N$-(2-bromophenylethyl)carbamoyl)

Erythromycin A, 6,11-di-O-methyl-4" -O( $N$-(2-chlorophenylethyl)carbamoyl)

Erythromycin A, 6,11-di-O-methyl-4"-O( $N$-(2-fluorophenylethyl)carbamoyl)

Erythromycin A, 6,11-di-O-methyl-4"-O( $N$-(3,4-dimethoxyphenylethyl)carbamoyl)

Erythromycin A, 6,11-di-O-methyl-4" -O( $N$-(3,4-methylenedioxyphenethyl)carbamoyl)

Erythromycin A, 6,11-di-O-methyl-4"-O( $N$-(3-butoxypropyl)carbamoyl)

Erythromycin A, 6,11-di-O-methyl-4" -O( $N$-(3-ethoxypropyl)carbamoyl)

Erythromycin A, 6,11-di-O-methyl-4" $-O$ ( $N$-(3-hydroxypropyl)carbamoyl)
Erythromycin A, 6,11-di-O-methyl-4" -O-

( $N$-(3-isopropoxypropyl)carbamoyl)

Erythromycin A, 6,11-di-O-methyl-4" -O(N-(3-methoxypropyl)carbamoyl)

Erythromycin A, 6,11-di-O-methyl-4" -O( $N$-(4-bromobenzyl)carbamoyl)

Erythromycin A, 6,11-di-O-methyl-4" -O(N-(4-chlorobenzyl)carbamoyl)

Erythromycin A, 6,11-di-O-methyl-4" -O( $N$-(4-fluorobenzyl)carbamoyl)

Erythromycin A, 6,11-di-O-methyl-4" -O( $N$-(4-fluorophenylethyl)carbamoyl)

Erythromycin A, 6,11-di-O-methyl-4" -O( $N$-(4-hydroxybenzyl)carbamoyl)

Erythromycin A, 6,11-di-O-methyl-4" $-O$ ( $N$-(4-hydroxyphenylethyl)carbamoyl)

Erythromycin A, 6,11-di-O-methyl-4" -O(N-(4-methoxybenzyl)carbamoyl)

Erythromycin A, 6,11-di-O-methyl-4" -O(N-(4-methoxyphenylethyl)carbamoyl)

Erythromycin A, 6,11-di-O-methyl-4"-O( $N$-(phenylethyl)carbamoyl)

Erythromycin A, 6,11-di-O-methyl-4" -O( $N$-benzylcarbamoyl)

Erythromycin A, 6,11-di-O-methyl-4" -O(N-butylcarbamoyl)

Erythromycin A, 6,11-di-O-methyl-4" -O$(N$-cyclohexylcarbamoyl)

Erythromycin A, 6,11-di-O-methyl-4"-O$(N$-furfurylcarbamoyl)

Erythromycin A, 6,11-di-O-methyl-4" -O$(N$-pentylcarbamoyl)

Erythromycin A, 6,11-di-O-methyl-4" -O(N-propylcarbamoyl)

Erythromycin, 8a-Aza-8a-homo, 4"-O-acyl derivatives

Etamycin

Everolimus

FG08

667

FK506

434,442

Flaviogeranin

379

$\begin{array}{ll}\text { Fluvirucin } & 415\end{array}$

Fosfomycin $\quad 657$

FR179642

FR235222

FR901379

$633,637,649$

Friulimicin

Fusidic acid

Fuzanin E, F, G, H, I

Gardine B, deoxyacta- $\quad 423$

GE2270 423

Ge23077 423

GE81112 423

Geldanamycin, 7-O-descarbamoyl-7-hydroxy 623

Gentamicin $\quad 442$

Gifhornenolone A, B 245

Gliotoxin $\quad 619$

Gliovirin $\quad 619$

Granaticin $\quad 442$ 
Griseoluteic acid

Griseolutein B

Haloduracin $\alpha, \beta$

Hatomarubigin C, E

Hericene D

Hericenone I

Homononactic acid

Hydroxy-3,4-dehydro-apo-8'-lycopene

(R)-16-Hydroxylinoleic acid

Hygrobafilomycin

Hygrolidin

ILS-920

Indazole-3-carbaldehyde

Isochromophilone I

Istamycin

Ivermectin

JBIR-14

JBIR-31

JBIR-58

JBIR-59

JBIR-65

JBIR-69

JBIR-74, 75

JBIR-79, 80

JBIR-81, 82

JBIR-83, 84

JBIR-86, 87

JBIR-88, 89

JBIR-89 derivative

JBIR-97, 98, 99

JBIR-100

Kanamycin

Kanamycin B

Ketoclomazone

KSA-9342

Lactarioline A, B

Lankacidin A

Laspartomycin

Lclaprim

Linezolid

Linoleic acid

Lonomycin

Lucensimycin

MAC13243

Macrolactin S, V

Macrotetrolide B, C, D, G

Mannopeptimycin A

Meleagrin

Meleagrin B, D, E

Methyl hydroxy-3,4-dehydro-apo- $8^{\prime}$ lycopenoate

Milbemycin

Minocycline

Monacolin J

Monacolin J derivatives
Monactin 524

Mutactimycin, 3-O-demethyl 442

Mutalomycin 486

NAI-107 423

NBRI16716A, B, C 319

NBRI17671 237

NBRI17671al 237

Nemadectin congener $\quad 171$

Nemonoxacin $\quad 423$

Neomycin $\quad 442$

Neomycin B 423

Neothramycin $\quad 381$

Neothramycin A, butyl 381

3-Nitro- $1 H$-indazole 303

Nocardicin A, B 442

Nocardithiocin $\quad 99$

Nonactic acid $\quad 524$

Nonactin 524

Nosokomycin A, B, C, D 151, 157

Novobiocin $\quad 492$

NVB302 423

NW-G01 231

NXL-103, $-104 \quad 423$

Nystatin 434

Nystatin $A_{1}, 28,29$-didehydro-

Nystatin $A_{1}, 28,29$-didehydro-, derivatives $\quad 55$

Ochrephilone $\quad 619$

Okilactomycin $\quad 415,619$

Oleandomycin 442

Oligomycin A 17

Ophiosetin 195

Orfamide 423

$\begin{array}{ll}\text { Oritavancin } & 423,717\end{array}$

Oxopropaline D, G 492

Paclitaxel $\quad 460$

Pactamycin $\quad 381$

Pactamycin, 7-deoxy 381

Paecilosetin 195

Paleic acid 519

$\begin{array}{ll}\text { Pargamicin A 279, } 339 & \end{array}$

Penicillin $\quad 442$

Penicillin N 442

Pentacecilide A, B, C, D 315

Pentamidine $\quad 381$

PF1022A $\quad 77$

Phellinin B, C 263

Phenazine $\quad 579$

Phenazine 5-oxide $\quad 579$

Phenazine, 1,6-Dimethoxy $\quad 579$

Phenazinomycin 579

Phenyl-2-bis-indolylmethane 303

(1R,2R)-1-phenylpropane-1,2-diol 575

$\begin{array}{ll}\text { Philipimycin } & 415,423\end{array}$

Piloquinone $\quad 619$

Piperacillin $\quad 423$

Platencin $\quad 415$

$\begin{array}{ll}\text { Platensimycin } & 415,423\end{array}$

Pleuromutilin $\quad 442$ 
Primycin 113

Pristinamycin 442

Promomycin 486

Proximicin $\quad 415$

Psychotrimine 685

Psychotrimine derivatives $\quad 685$

Pyrenocine A, B, I 559

Pyridine monothiocarboxylic acid analogs 255

PZ-601 423

RAD001 434

Radezolid 423

Rakicidin A, B, C, D 563

Ramoplanin 423

Rapamycin 434, 442

Rapamycin, 32-desmethoxy 434

Rapamycin, 32-desmethyl 434

Rapamycin, 4-hydroxyprolyl, 4-hydroxyproline 434

Rapamycin, 4-hydroxyprolyl-26-demethoxy 434

Rapamycin, 4-thia 434

Rapamycin, prolyl 434

Rapmycin, 9-deoxo-5-sulfoxy $\quad 434$

Retamycin 442

Rifampicin 23

Rifampicin, $2^{\prime}-N$-hydroxy-4-oxo 23

Rifampicin, $2^{\prime}-N$-oxide $\quad 23$

Rifampicin, $6^{\prime}-N$-oxide $\quad 23$

Rifampin 51

Rifamycin $\quad 442$

Roquefortine H, I $\quad 165$

Roridin E 539

Rosamicin, 23-O-mycinosyl-20-dihydro- 325

Roselipin 3A, 3B, 3C 3D, 3E 119

Russulfoen $\quad 575$

RWJ-416457 423

$\begin{array}{ll}\text { Ryanodine } & 77\end{array}$

$\begin{array}{lr}\text { Salinosporamide A } & 415\end{array}$

Sansanmycin F, G $\quad 143$

SF2583A, B 619

Sinefungin $\quad 673$

Sinefungin V, dehydro $\quad 673$

Sinefungin VA $\quad 673$

Sinefungin, dehydro 673

SMTP-10, -11, -12, -13, -14, -15, -16, -33, -38, -40, -42 589

SNF4435C 601

Spectinabilin $\quad 601$

Spinetoram 101

Spinosyn

Spinosyn A, B, C, D, E, F, G, H, J, K, L, $\alpha 1, \delta 1$
Spinosyn A aglycone

101

Spinosyn J, 3'-O-ethyl-5,6-dihydro- 101

Spinosyn L, 3'-O-ethyl- $\quad 101$

Spiramycin $\quad 442$

Staurosporin $\quad 442$

Streptomycin 442

Streptomycin, 5'-hydroxy 442

$\begin{array}{ll}\text { Suramin } & 381,579\end{array}$

Surugapyrone A $\quad 365$

Tazobactam 423

Teicoplanin $\quad 279$

Telavancin $\quad 423$

Teleocidin $\mathrm{A}_{1}$

Temsirolimus $\quad 434$

Terpeptin $\quad 389$

Tetracenomycin $\quad 442$

Tetracycline 279, 442, 693

Tetranactin 524

Thiane, 1,4-di 303

Thiazomycin $\quad 423$

Thienamycin $\quad 135$

Thiocillin 423

$\begin{array}{ll}\text { Thiolutin } & 619\end{array}$

Thiomuracin $\quad 423$

Thuggacin A 423

Tigecycline 29, 51, 693

Torezolid 423

Trichosporin B-VIIa, -VIIb 331

$7 \alpha, 8 \alpha, 13$-Trihydroxy-marasm-5-oic acid $\gamma$-lactone $\quad 575$

Trinactin 524

Tumescenamide A, B 549

Turbomycin A, B $\quad 415$

Vancomycin

Verticilide

Walkmycin A, B, C $\quad 89$

Walrycin A, B 127

WAY-125286, -24688 434

WCK-771

WYE-592 434

Xanthoradone A, B, C $\quad 329$

Zabofloxacin $\quad 423$

$97518 \quad 423$

$2754 \mathrm{~A}-721$ 